\title{
Eco friendly synthesis, spectroscopic investigation of copper and its oxide nanoparticles with excellent electrical conductivity
}

\begin{abstract}
A facile and rapid route for the synthesis of uniform Nano copper and its oxide $(\mathrm{CuO}$ NPs) by a microwave assisted method is demonstrated. The copper and its oxide nanoparticles (CuO NPs) prepared exhibit excellent conductivity, which is comparable with electrospun copper nanofibers. Among the various metal nanoparticles, Copper and its oxide nanoparticles (CuONPs) have attracted considerable attention because copper is one of the most important in modern technology and is readily available. Copper Nanoparticles have been much attractive because it is easily available, cost effective and conducting nature. Copper and its oxide Nanoparticles were synthesized using Copper (II) succinate by microwave conventional method. Copper and its oxide Nanoparticles so obtained were characterized by UV- Visible spectroscopy, Fourier Transform Infrared Spectroscopy and X-Ray diffraction analysis. X-Ray diffraction analysis proved the formation of Copper and its oxide Nanoparticles. SEM and AFM analyses showed the presence of Nanoparticles.
\end{abstract}

Keywords: copper sulfate, uv visible, xrd, ft-ir, AFM
Volume 3 Issue I - 2017

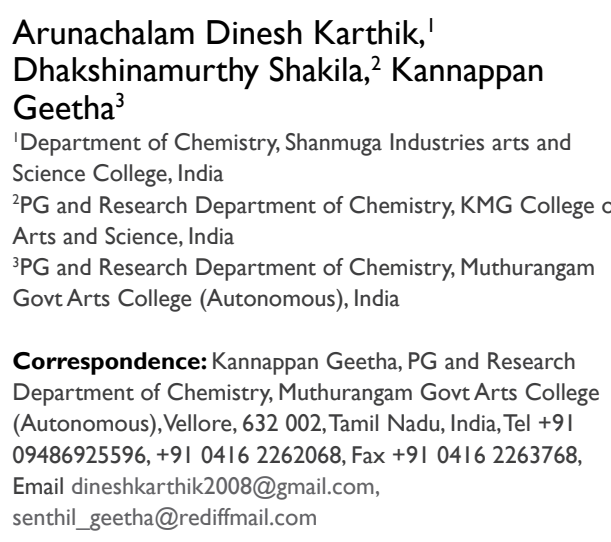

Received: August 18,2017 || Published: November 06, 2017

\section{Introduction}

Metal Nanoparticles have attracted considerable attention because of their excellent electronic and optical properties. They have been used in many fields, such as displays, solar cells, touch-screens, sensors, and electromagnetic interference shielding. ${ }^{1}$ However, for most of these applications, metal Nanoparticles with high electrical conductance are required. Compared with various other metals, copper is more abundant and less expensive, while having favourable mechanical, electrical, and optical properties. ${ }^{2}$ Copper Nanoparticles have thus been suggested as a low cost alternative to indium tin oxide (ITO), investigated extensively and compared against conductive polymers, carbon nanotubes, graphenes and $\mathrm{Ag}$ nanowires..$^{3-6} \mathrm{To}$ date, various methods have been developed for synthesizing Copper and its oxide Nanoparticles (CuO NPs), including electrochemical deposition, electrospinning, template processes, hydrothermal reduction and reverse micellar systems. ${ }^{7-12}$ On the other hand, the microwave-assisted synthesis. ${ }^{13,14}$ which is generally quite fast, simple and efficient in energy, has been developed and is widely used in various fields such as molecular sieve preparation, radiopharmaceuticals, the preparation of inorganic complexes and oxide, organic reactions, plasma chemistry, analytical chemistry and catalysis. Generally speaking, the power, heating frequency and on/ off irradiation cycles are the main heating parameters of a microwave oven and each of them may have a great effect on the structure and properties of the products. To the best of our knowledge, although such a heating method has been a focus of research, most of previous reports were limited to fixed working conditions of the microwave oven. ${ }^{15-20}$ and there is no comprehensive report addressing the effects of these heating parameters on the synthesis of nanomaterials. ${ }^{21-23}$ Furthermore, there are few reports on synthesizing the Copper and its oxide Nanoparticles without template at mild condition by the use of microwave method. Here, we report a simple microwave method to fabricate well aligned Copper and its oxide Nanoparticles (CuO NPs) on a Copper surface without any template and surfactant. ${ }^{24-26}$

\section{Experiment}

\section{Materials}

All the chemicals, reagents used in our experiments were of analytical grade and were used as received without further purification. Succinic acid, Copper sulphate and $\mathrm{NaOH}$ were purchased from Aldrich and were used as received, hexane SDF and ethanol from distillery.

\section{Synthesis of the Copper(II) succinate precursor}

The $\mathrm{CuSO}_{4} \cdot 5 \mathrm{H}_{2} \mathrm{O}(2 \mathrm{mmol})$ was dissolved in $10 \mathrm{~mL}$ of distilled water to form a homogeneous solution. A stoichiometric amount of sodium hydroxide $(\mathrm{NaOH})$ and succinic acid were dissolved in distilled water. The sodium succinate thus formed was drop wise added into the above solution under magnetic stirring. The solution was stirred for about $30 \mathrm{~min}$ and a green precipitate was obtained which was centrifuged and washed with ethanol several times. The product was dried. The Copper (II) succinate was characterized various spectral techniques. ${ }^{27}$

\section{Synthesis of the copper and its oxide nanoparticles}

Copper and its oxide nanoparticles were synthesized by a domestic microwave method in the presence of Copper (II) succinate. The Copper (II) succinate placed in a domestic microwave. Finally, the reaction system was heat treated at $473 \mathrm{~K}$ for $2 \mathrm{~h}$. Copper and its oxide Nanoparticles were obtained black powder (Scheme $1 \& 2$ ). 


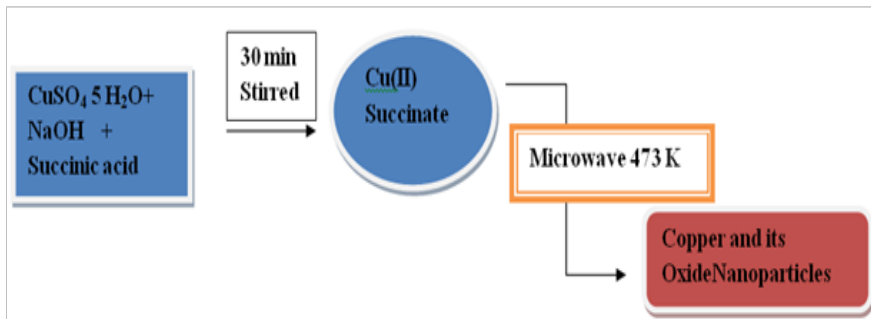

Scheme I Schematic diagram of the formation of Copper and its oxide Nanoparticles.

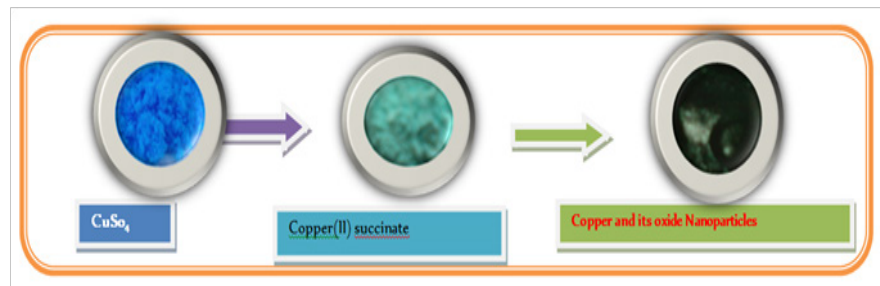

Scheme 2 Photographic picture of the formation of Copper (II) succinate and Copper \& its oxide Nanoparticles.

\section{Results and discussion}

\section{UV Visible Spectra}

Copper Nanoparticles typically exhibit $\sim 500 \mathrm{~nm}$. However the Copper (II) succinate show an absorption peak at 264,372 and 568nm (Figure 1) and also Copper and its oxide Nanoparticles synthesized here show an absorption peak at $450 \mathrm{~nm}$ which can be assigned to the absorption of Nanoparticles of copper. Tauc plot for synthesized Nanoparticles is shown in Figure 1. This peak can be assigned to the absorption of Nanoparticles of copper. A modest blue shift of the absorption edge relative to that of the bulk copper powder is observed. This observation alludes to the size effect of copper and its oxide Nanoparticles.

\section{FT - IR measurement}

FT-IR spectroscopy is a useful tool to understand the functional group of any organic molecule Figure 2A shows IR spectrum of Copper (II) Succinate precursor. Figure 2B shows IR spectrum of copper Nanoparticles. A twin peak at $621 \mathrm{~cm}^{-1} \& 680 \mathrm{~cm}^{-1}$ indicated the $\mathrm{Cu}-\mathrm{O}$ Stretching vibration. The metal salt $(\mathrm{Cu}-\mathrm{O}-\mathrm{C})$ Peak appeared at $1192 \mathrm{~cm}^{-1}$. A Peak at $3541 \mathrm{~cm}^{-1}$ indicates $\mathrm{OH}$ stretching of the water in the precursor which disappeared in the Nanoparticles. A Peak at $1637 \mathrm{~cm}-1$ indicates metal carbonyl $(\mathrm{C}=\mathrm{O})$ of the precursor which disappeared in the Nanoparticles B3. ${ }^{28-30}$
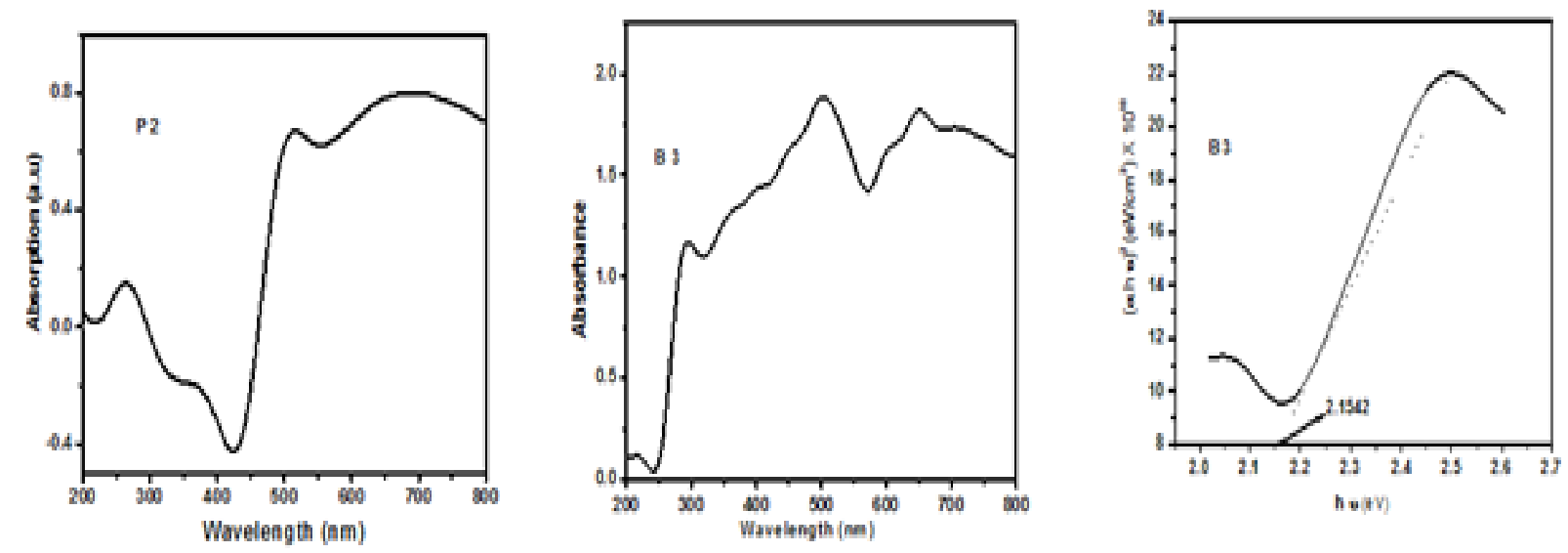

Figure I UV-Visible spectrum of Precursor P2, Copper and its oxide Nanoparticles of B3 \& tauc plot of B3.
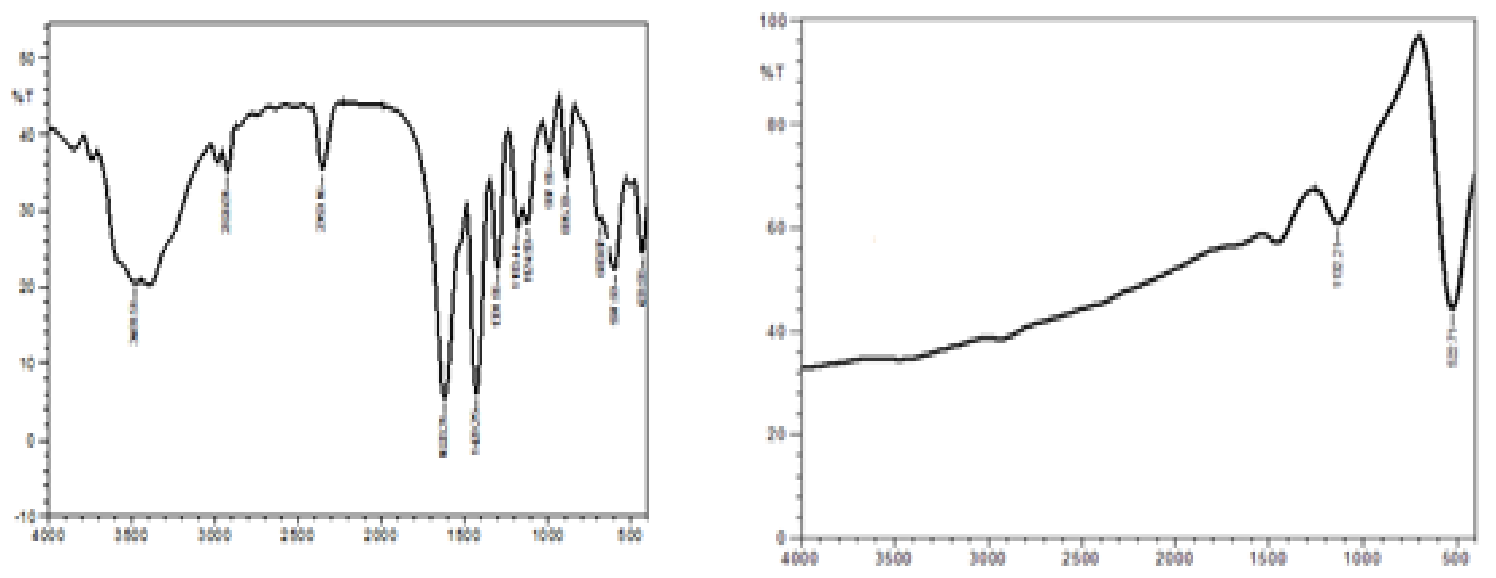

Figure 2A-B: FT-IR spectrum of P2 and copper Nanoparticles of B3 


\section{X-Ray diffraction studies}

XRD pattern of Copper and its oxide nanoparticles deposited at $300^{\circ} \mathrm{C}$ under nitrogen environment with a collecting angle varying from 20 to $80^{\circ}$ and the usual copper $K \alpha 1$ radiation $\lambda=1.5406 \AA$ is shown in Figure $3 \&$ Table 1. The hkl values (111), (200), (220), $2 \theta$ values 48.96, 53.67, and 68.24 and corresponding d values, 2.073, 1.796 , and $1.274 \AA$ are used for determining lattice parameter which came to be $3.01,4.01$, and $8.00 \AA$, respectively using standard formula (Lattice $=\mathrm{d} \sqrt{ }$ h.k.1). The relative peak intensities and the position of diffraction peaks in the XRD pattern are very similar to those reported in the JCPDS standard data 40836 and others. The Table 2 shows particle size of Copper and its oxide nanoparticles. The position and the integral width of $x$-ray diffraction peaks are determined to identify the crystalline phase which is found to be face centered cubic. ${ }^{31}$

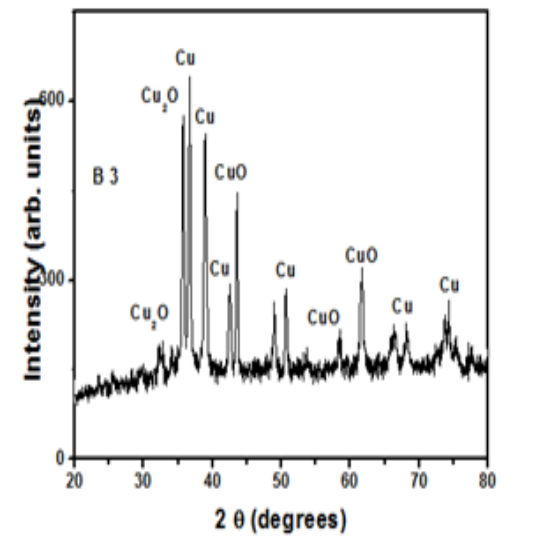

Figure 3 XRD pattern of the copper and its oxide nanoparticles.

Table I Peak indexing from d-spacing

\begin{tabular}{lllll}
\hline $\mathbf{2 \theta}$ & $\mathbf{d}$ & $\mathbf{1 0 0 0 / \mathbf { d } ^ { 2 }}$ & $\left(\mathbf{1 0 0 0 / \mathbf { d } ^ { 2 } ) / 7 7 . 3 2}\right.$ & hkl \\
\hline 48.96 & 2.073 & 232.07 & $3.0 \mathrm{I}$ & $\mathrm{III}$ \\
53.67 & 1.796 & 310.02 & 4.01 & 200 \\
68.24 & 1.274 & $616.1 \mathrm{I}$ & 8 & 220 \\
\hline
\end{tabular}

Particle size calculation: The existence of sharp peaks indicates that the synthesized nanoparticles are polycrystalline in nature. ${ }^{31}$ One can calculate the values of average crystallite size Debye - Scherer formula (D) from eq (1), d-Spacing the value of $d$ (the inter planar spacing between the atoms) is calculated from equation (2) and microstrain (ع) from equation (3) formation XRD spectrum using the following equations:

$$
[D=0.9 \lambda / \beta \cos \theta]
$$

Bragg's Law: $2 d \sin \theta-n \lambda$

$$
d=\lambda \quad(\mathrm{n}=1)
$$

$2 \operatorname{Sin} \theta$

Wavelength $\lambda=1.5418 \AA$ for $\mathrm{Cu} \mathrm{Ka}$

$\varepsilon=\beta / 4 \tan \theta$ (3)

The calculated d-spacing and micro strain details are in Table 2.

\section{Cyclic voltametry}

Figure 4 shows cyclic voltammogram (CV) the instructor will familiarize the students with the operation of the computer program interfaced to the $\mathrm{CV}$ and the proper procedure for introducing and removing solutions from the cell. Set the Copper Nanoparticles was recorded in DMSO with $0.1 \mathrm{M}$ tetrabutyl ammonium perchlorate as supporting electrolyte in the initial potential $+2 \mathrm{~V}$ and $-2 \mathrm{~V}$. The scan should be initiated in a positive direction with a scan rate of $50 \mathrm{mV} / \mathrm{s}$ with a conventional three electrode system composed of a platinum auxiliary, Glassy carbon working electrode and Calomel (Saturated $\mathrm{KCl}$ ) reference electrode. The reductive peaks correspond to $\mathrm{Cu}$ (II)/ $\mathrm{Cu}$ (I) and $\mathrm{Cu}(0)$ i.e $\mathrm{CuO} \rightarrow \mathrm{Cu}_{2} \mathrm{O} \rightarrow \mathrm{Cu}$. After the deoxygenating is complete switch on the working electrode and wait for approximately $10 \mathrm{~s}$ until the current has reached a constant value. Initiate the potential scan and a background CV of the supporting electrolyte is obtained. This scan with a wide potential range provides the operational limits of potential at which the experiment can be run, i.e. the potential window of the electrolyte. The redox potential values obtained from the cyclic voltammogram for the Copper and its oxide Nanoparticles were quasi-reversible and the electron flow was sluggish (Table 3). Data from Cyclic voltammetric measurements; $\mathrm{E}_{1 / 2}$ is calculated as average of anodic (Epa) and cathodic (Epc) peak potentials $\mathrm{E}_{1 / 2}=1 / 2(\mathrm{Epa}+\mathrm{Epc})$

Table 2 The particle size of B3

\begin{tabular}{llllllll}
\hline $\begin{array}{l}\text { 20 of the } \\
\text { intense peak } \\
(\mathbf{d e g})\end{array}$ & $\begin{array}{l}\boldsymbol{\theta} \text { of the } \\
\text { intense } \\
\text { peak }(\mathbf{d e g})\end{array}$ & $\begin{array}{l}\text { FWHM of } \\
\text { intense peak }(\boldsymbol{\beta}) \\
\text { radians }\end{array}$ & $\begin{array}{l}\text { Size of the } \\
\text { particle } \\
(\mathbf{D}) \mathbf{n m}\end{array}$ & $\begin{array}{l}\mathbf{d} \\
\text { spacing } \\
\mathbf{n m}\end{array}$ & Plane & Material & Microstrain \\
\hline 32.68 & 16.34 & 0.1378 & 60.4 & 2.7401 & -202 & $\mathrm{CuO}$ & 0.1175 \\
35.75 & 17.875 & 0.1574 & 53.3 & 2.5115 & -110 & $\mathrm{Cu}_{2} \mathrm{O}$ & 0.122 \\
38.98 & 19.49 & 0.2362 & 35.7 & 2.31055 & -111 & $\mathrm{Cu}$ & 0.1668 \\
48.96 & 24.48 & 0.1968 & 44.5 & 1.8604 & -200 & $\mathrm{Cu}$ & 0.108 \\
53.67 & 26.835 & 0.1378 & 65 & 1.7077 & -20 & $\mathrm{CuO}$ & 0.0681 \\
58.49 & 39.245 & 0.2166 & 42.1 & 1.578 & -200 & $\mathrm{Cu}$ & 0.0967 \\
61.72 & 30.86 & 0.1378 & 67.5 & 1.5029 & -200 & $\mathrm{Cu}$ & 0.576 \\
66.44 & 33.22 & 0.1368 & 48.4 & 1.4072 & -200 & $\mathrm{Cu}$ & 0.0751 \\
68.24 & 34.12 & 0.168 & 57.3 & 1.3743 & -20 & $\mathrm{CuO}$ & 0.06198 \\
\hline
\end{tabular}



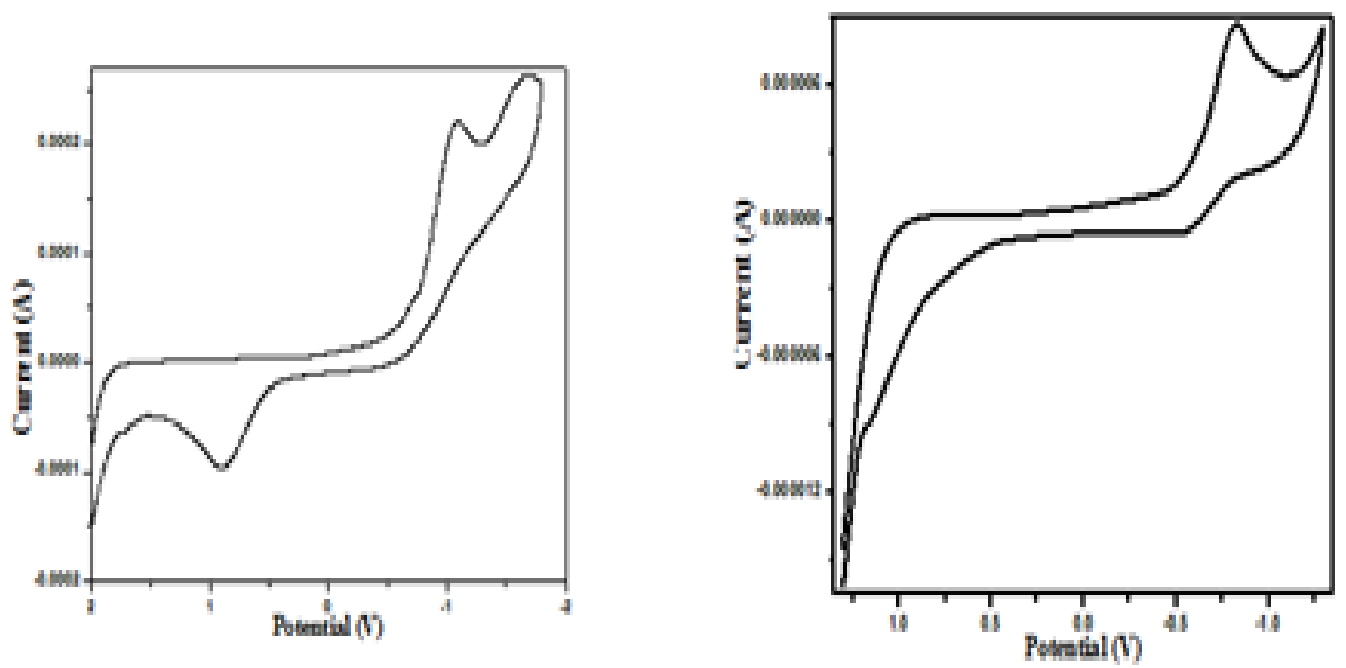

Figure 4 Cyclic voltammogram of the $\mathrm{P} 3$ and $\mathrm{B} 3$.

Table 3 Redox Potentials of Copper and its oxide nanoparticles in DMSO solution at 298K

\begin{tabular}{llllllll}
\hline Compound & $\mathrm{Epc}(\mathrm{V})$ & $\mathrm{Epa}(\mathrm{V})$ & $\Delta \mathrm{E}(\mathrm{V})$ & $\mathrm{EI} / 2$ & $\mathbf{i p c}(\boldsymbol{\mu A})$ & $\mathbf{i p a}(\boldsymbol{\mu A})$ & $\mathrm{ipa} / \mathrm{ipc}$ \\
\hline P2 & -1.0901 & 0.9035 & 1.9936 & 0.0933 & $1.6120 \times 10-4$ & $7.782 \times 10-5$ & 0.4827 \\
B3 & -0.8354 & -0.5376 & 0.2978 & -0.6865 & $7.993 \times 10-6$ & $2.162 \times 10-6$ & 0.2704
\end{tabular}

\section{SEM \& AFM analysis for copper and its oxide nanopar- ticles}

The morphology of the product is examined by SEM. Figure 5 depicts the SEM pictures of a sample of Copper and its oxide Nanoparticles. From the micrograph, it was observed that the Nanoparticles are agglomerated. The Atomic force microscopic (AFM) photographs of the product are given in Figure 6. These sizes are nearly cubic (fcc). The area roughness and RMS are in the range 160 to $487 \mathrm{~nm}$ which indicates its conducting nature. We may consider that when the reaction was carried out at $473 \mathrm{~K}$, most organic molecules decomposed. Copper and its oxide nano have rod like, loose solid morphologies.
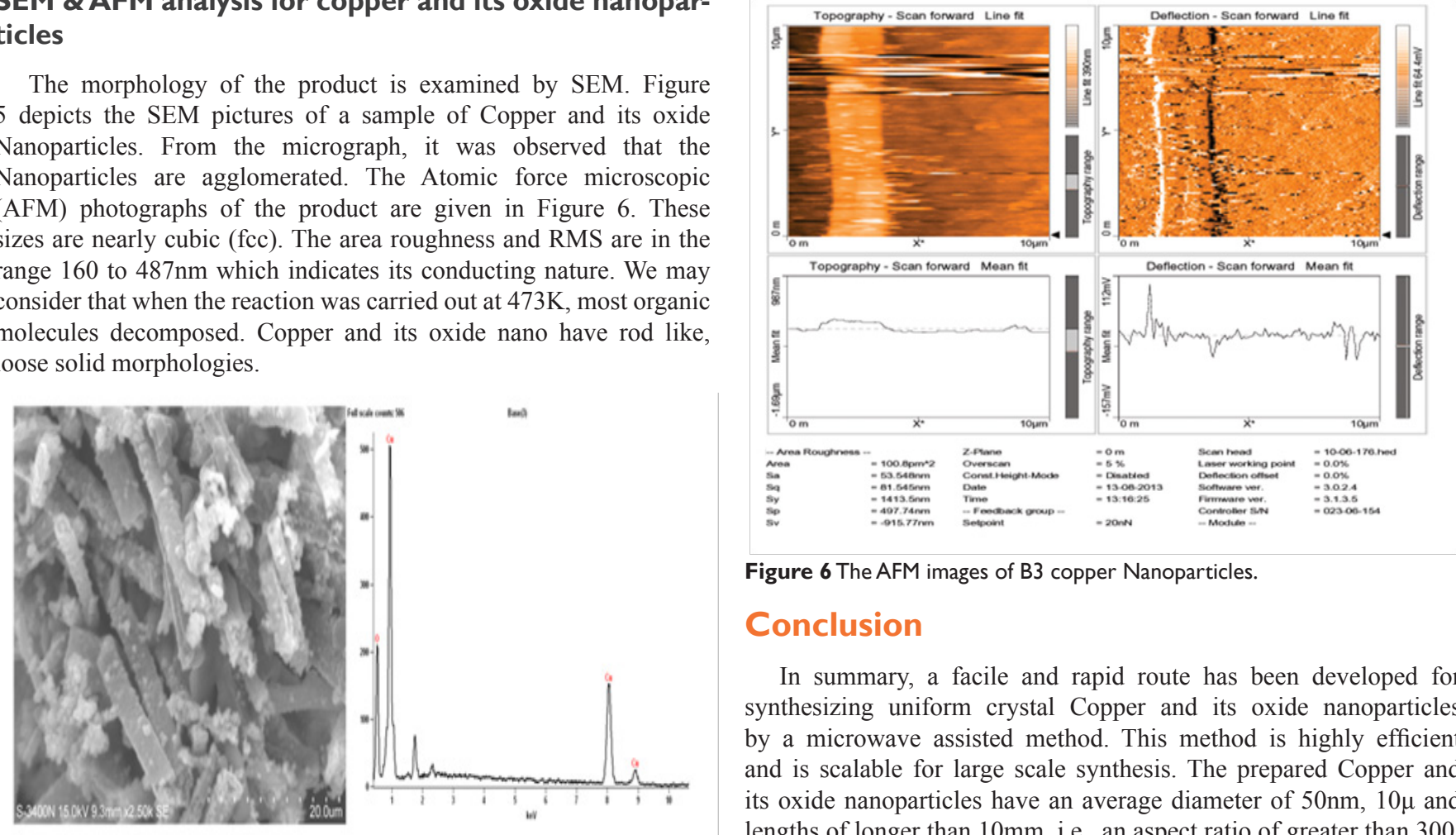

Figure 6 The AFM images of B3 copper Nanoparticles.

\section{Conclusion}

In summary, a facile and rapid route has been developed for synthesizing uniform crystal Copper and its oxide nanoparticles by a microwave assisted method. This method is highly efficient and is scalable for large scale synthesis. The prepared Copper and its oxide nanoparticles have an average diameter of $50 \mathrm{~nm}, 10 \mu$ and lengths of longer than $10 \mathrm{~mm}$, i.e., an aspect ratio of greater than 300 . Moreover, the Copper and its oxide nanoparticles show outstanding conductivity, comparable with electrospun copper nanofibers. Our experimental results demonstrate that this novel and simple route can 
produce high quality crystalline Copper and its oxide Nanoparticles of nanostructures.

\section{Acknowledgements}

Authors are grateful thank to Principal, Muthurangam Govt. Arts College (Autonomous), Vellore, for providing facilities to undertake this work. We also thank Center for nanotechnology, VIT Vellore for AFM analysis.

\section{Conflict of interest}

The author declares no conflict of interest.

\section{References}

1. Moulton B, Zaworotko MJ. From molecules to crystal engineering: supramolecular isomerism and polymorphism in network solids. Chem Rev. 2001;101(6):1629-1658.

2. Kitagawa S, Kitaura R, Noro SI. Functional porous coordination polymers. Angew Chem Int Ed Engl. 2004;43(18):2334-2375.

3. Janiak CJ. Engineering coordination polymers towards applications, Dalton Trans. 2003;14:2781.

4. James SL. Metal-organic frameworks. Chem Soc Rev. 2003;32(5):276288.

5. Carlucci L, Ciani G, Proserpio D. Polycatenation, polythreading and polyknotting in coordination network chemistry. Coord Chem Rev. 2003;246:247-289.

6. Wong KT, Lehn JM, Peng SM, et al. Nanoscale molecular organometallo wires containing diruthenium cores. Chem Commun. 2000;22:2259.

7. Bunn AG, Carroll PJ, Wayland BB. Coordination polymers of tetracyanoethylene with metal hexafluoroacetylacetonates: formation and $\mathrm{X}$ ray crystal structure. Inorg Chem. 1992;31(7):1297-1299.

8. Caneschi A, Gatteschi D, Lalioti N, et al. Cobalt(II)-nitronyl nitroxide chains as molecular magnetic nanowires. Angew Chem Int Ed Engl. 2001;40(9):1760-1763.

9. Tanatani A, Moi MJ, Moore JS. Chain length-dependent affinity of helical foldamers for a rodlike guest. J Am Chem Soc. 2001;123(8):17921793.

10. Fujita M, Kwon YJ, Washizu S, et al. Preparation, clathration ability, and catalysis of a two-dimensional square network material composed of cadmium (II) and 440-bipyridine. J Am Chem Soc. 1994;116(3):11511152 .

11. Jin R, Cao Y, Mirkin CA, et al. Photoinduced conversion of silver nanospheres to nanoprisms, Science. 2001;294(5548):1901-1903.

12. Koch CC. Nanostructured materials: processing, properties and applications. Norwich, UK: William Andrews; 2002. 397 p.

13. Jun YW, Seoa JW, Oh SJ, et al. Recent advances in the shape control of inorganic nano-building blocks. Coord Chem Rev. 2005;249(1718):1766-1775.

14. Kim F, Connor S, Song H, et al. Platonic gold nanocrystals. Angew Chem Int Ed Engl. 2004;43(28):3673-3677.
15. Lv S, Li P, Sheng J, Sun W. Synthesis of single-crystalline BaCO3 nanostructures with different morphologies via a simple PVP-assisted method. Mater Lett. 2007;61(21):4250-4254.

16. Li Y, Liang J, Tao Z, et al. CuO particles and plates: synthesis and gas-sensor application. Mater Res Bull. 2008;43(8-9):2380-2385.

17. Zhang $\mathrm{H}$, Zhang $\mathrm{M}$. Synthesis of $\mathrm{CuO}$ nanocrystalline and their application as electrode materials for capacitors. Mater Chem Phys. 2008;108(23):184-187.

18. Arbuzova T, Gizhevskii B, Naumov S, et al. Temporal changes in magnetic properties of high-density $\mathrm{CuO}$ nanoceramics. J Magn Magn Mater. 2003;258:342-344.

19. Gao P, Chen $\mathrm{Y}, \mathrm{Lu} \mathrm{H}$, et al. Synthesis of $\mathrm{CuO}$ nanoribbon arrays with noticeable electrochemical hydrogen storage ability by a simple precursor dehydration route at lower temperature. Int $J$ Hydrogen Energ. 2009;34(7):3065-3069.

20. Maruyama T. Copper oxide thin films prepared by chemical vapor deposition from copper dipivaloylmethanate. Sol Energ Mater Sol. 1998;56(1):85-92.

21. Yao M, Tang Y, Zhang L, et al. Photocatalytic activity of $\mathrm{CuO}$ towards HER in catalyst from oxalic acid solution under simulated sunlight irradiation. Trans Nonferrous Met Soc China. 2010;20(10):1944-1949.

22. Chen JT, Zhang F, Wang J, et al. CuO nanowires synthesized by thermal oxidation route. J Alloys Compd. 2008;454(1-2):268-273.

23. Yu L, Zang G, Wu Y, et al. Cupric oxide nanoflowers synthesized with a simple solution route and their field emission. J Cryst Growth. 2008;310(12):3125-3130.

24. Zhu J, Bi H, Wang Y, et al. Synthesis of flower-like $\mathrm{CuO}$ nanostructures via a simple hydrolysis route. Mater Lett. 2007;61(30):5236-5238.

25. Dinesh karthik A, Geetha K. Synthesis and characterization of copper and copper oxide nanoparticles by thermal decomposition method. Int $J$ NanoDimens. 2014;5(4):321-327.

26. Dinesh karthik A, Geetha K. Synthesis of copper precursor, copper and its oxide nanoparticles by green chemical reduction method and its antimicrobial activity. Journal of Applied Pharmaceutical Science. 2013;3(5):016-021.

27. Zhang $\mathrm{H}$, Li S, Ma X, et al. Controllable growth of dendrite-like $\mathrm{CuO}$ nanostructures by ethylene glycol assisted hydrothermal process. Mater Res Bull. 2008;43(5):1291-1296.

28. Nakamoto K. Infrared and raman spectra of inorganic and coordination compounds. 5th ed. New York, USA: John Wiley and Sons; 1997.

29. Tenga F, Yao W, Zheng Y, et al. Synthesis of flower-like CuO nanostructures as a sensitive sensor for catalysis. Sensors Actuat B. 2008;134(2):761-768.

30. Song X, Yu H, Sun S. Single-crystalline CuO nanobelts fabricated by a convenient route. J Colloid Interf Sci. 2005;289(2):588-591.

31. Cullity Addison BD. (1978) Elements of X-Ray Diffraction. Wesley Pub co USA, p. 4-47. 